\title{
Study of $Z^{0} / \gamma^{*}+$ Jet via Electron Decay Mode at $\sqrt{s}=7 \mathrm{TeV}$ in CMS @ LHC
}

\author{
U.Bhawandeep, Suman B.Beri \\ Panjab University, Chandigarh 160 014, India \\ (on behalf of CMS Collaboration) \\ E-mail: bhawanuppal11@gmail.com
}

\begin{abstract}
The area-normalized angular distributions in events containing a $Z^{0}$ boson and a jet, using the electron decay mode are presented. The data samples correspond to $5 \mathrm{fb}^{-1}$ of proton-proton collisions at $\sqrt{s}=7 \mathrm{TeV}$, collected by the CMS detector. Events in which there is a $\mathrm{Z}$ boson and at least one jet, with a jet transverse momentum threshold of $30 \mathrm{GeV} / \mathrm{c}$ and absolute jet rapidity less than 2.4 , are selected for this analysis. We compare our measurements with a next-to-leading-order perturbative QCD calculation and two generators that combine tree-level matrix element calculations with parton showers.
\end{abstract}

\section{Introduction}

The measurement of the angular distributions has been a tool for understanding the structure and interactions of matter from the time of Rutherford's first scattering experiments. At the Large Hadron Collider (LHC), copious amounts of prompt photons and Z bosons (collectively called V) in association with jets are produced. The measurement of the rapidity distributions of $\mathrm{Z}+$ jet events is necessary for the characterization of the Higgs boson properties, and provides an important test of the modeling of these properties in theory calculations. For Z boson decays to electron and muon pairs, the signal is nearly background-free, and triggering is very efficient. From a theoretical point of view, the presence of the electroweak vertex makes the perturbative calculation of dynamical quantities more stable, and next-to-leading order (NLO) perturbative quantum chromodynamics (pQCD) calculations exist for $\mathrm{Z}+$ up to 4 jets [1] and photon+jet production [2, 3].

The rapidity of a particle is defined as $Y=1 / 2 \log ((E+p z) /(E-p z))$, where $\mathrm{E}$ is the energy and $p_{z}$ is the momentum component along the direction of one of the proton beams. If the $\mathrm{V}$ and the jet are the only objects in the event, the polar scattering angle $\left(\theta^{*}\right)$ is defined with respect to the incident proton direction in the two-object center of momentum (COM) reference frame, and can be written in terms of the measured quantities $Y_{V}$ and $Y_{\text {jet }}$ as $\cos \theta^{*}=\tanh \left(Y_{\text {dif }}\right) / \beta^{*}$, where $\beta^{*}$ is the speed of the $\mathrm{V}$, and $Y_{\text {dif }}=\left|Y_{V}-Y_{\text {jet }}\right| / 2$. The quantity $Y_{\text {sum }}=\left|Y_{V}+Y_{\text {jet }}\right| / 2$ approximates the rapidity boost from laboratory to the COM reference frame. In the laboratory frame, $Y_{V}$ and $Y_{j e t}$ are highly correlated because there is usually a relatively high momentum quark interacting with a low momentum gluon or anti-quark. The transformed rapidity relations $\left(Y_{\text {sum }}, Y_{\text {dif }}\right)$ are effectively a rotation in phase space of the system $\left(Y_{V}, Y_{j e t}\right)$, which results in the two approximately uncorrelated quantities. At leading-order (LO), matrix element calculations of $V+$ jet events have asymptotic small angle $\cos \theta^{*}$ distributions which vary in proportion to $1 /\left(1-\left|\cos \theta^{*}\right|\right)$, while the di-jet spectrum is proportional to $1 /\left(1-\left|\cos \theta^{*}\right|\right)^{2}$. The difference is due to the spin of their respective propagators. Theory predictions of all the four $\mathrm{Y}$ distributions: $Y_{V}, Y_{\text {jet }}, Y_{\text {sum }}$ and $Y_{\text {dif }}$, are compared with data taken by the Compact Muon Solenoid (CMS) detector. 
Table 1. The data sets used in this analysis, the corresponding numbers of events, and run ranges.

\begin{tabular}{|l|cc|c|}
\hline Name & Run-Range & nEvents \\
\hline /DoubleElectron - Run2011A -08 Nov2011 - v1 & 160329 & 175770 & 49853042 \\
/DoubleElectron - Run2011B - 19Nov2011 - v1 & 175832 & 180296 & 15618141 \\
\hline
\end{tabular}

Table 2. MC Datasets and corresponding products of cross-section times branching ratio (in units of $\mathrm{pb}$ ) used in the analysis.

\begin{tabular}{|c|c|}
\hline Sample & $\sigma \times B(p b)$ \\
\hline /DYJetsToLL_TuneZ2_M - 50_7TeV - madgraph - tauola/Fall11 - PU_S6_START44_V9B - v1/AODSIM & $3048(\mathrm{NNLO})$ \\
\hline /DYToLL_M - 50_1jEnh2_2jEnh35_3jEnh40_4jEnh50_7TeV - sherpa/Fall11 - PU_S6_START44_V9B - v1/AODSIM & 3048 (NNLO) \\
\hline /WJetsToLNu_TuneZ2_7TeV - madgraph - tauola/Fall11 - PU_S6_START44_V5 - v1/AODSIM & $31314(\mathrm{NNLO})$ \\
\hline /TTJets_TuneZ2_7TeV - madgraph - tauola/Fall11 - PU_S6_START44_V9B - v1/AODSIM & $157.5(\mathrm{NLO})$ \\
\hline /QCD_Pt - 20to30_BCtoE_TuneZ2_7TeV - pythia6/Fall11 - PU_S6_START44_V9B - v1/AODSIM & $236100000 * 0.00059$ \\
\hline /QCD_Pt - 30to80_BCtoE_TuneZ2_7TeV - pythia6/Fall11 - PU_S6START44_V5 - v1/AODSIM & $59440000 * 0.00242$ \\
\hline /QCD_Pt - 80to170_BCtoE_TuneZ2_7TeV - pythia/Fall11 - PU_S6 - START44_V5 - v1/AODSIM & $898200.0 * 0.0105$ \\
\hline /QCD_Pt - 20to30_EMEnriched_TuneZ2_7TeV - pythia6/Fall11 - PU_S6_START42_V14B - v1/AODSIM & $236100000 * 0.0106$ \\
\hline /QCD_Pt - 30to80_EMEnriched_TuneZ2_7TeV - pythia/Fall11 - PU_S6 - ST ART44_V5 - v1/AODSIM & $59440000 * 0.061$ \\
\hline /QCD_Pt - 80to170_EMEnriched_TuneZ2_7TeV - pythia6/Fall11 - PU_S6_START42_V14B - v2/AODSI & $898200.0 * 0.159$ \\
\hline
\end{tabular}

The paper is organised as follows: In Section 2, we briefly discuss some relevant details of detector. Section 3 lists the data and simulated samples used in this analysis while Section 4 describes our selection criteria. Results for Data and MC comparison are discussed in Section 5. We conclude in Section 6.

\section{CMS Detector}

A counterclockwise coordinate system is used in CMS, with the origin at the nominal interaction point, the x-axis pointing to the center of the LHC ring, the y-axis pointing up (perpendicular to the LHC plane), and the z-axis along the anti-clockwise beam direction. The polar angle $\theta$ is measured from the positive z-axis and the azimuthal angle $\phi$ is measured in the $\mathrm{x}$-y plane. Pseudo-rapidity, which is given by $\eta=\ln [\tan (\theta / 2)]$, is used for acceptance requirements. The central feature of the CMS apparatus is a superconducting solenoid of $6 \mathrm{~m}$ internal diameter, providing a magnetic field of $3.8 \mathrm{~T}$. Within the field volume are a silicon pixel and strip tracker, an electromagnetic calorimeter, and a brass/scintillator hadron calorimeter. Muons are detected in gas-ionization detectors embedded in the steel return yoke. In addition to the barrel and endcap detectors, CMS has extensive forward calorimetry. A more detailed description of CMS is given in [4].

\section{Data and MC Samples}

The data samples for this analysis are collected with the di-electron triggers and are listed in Table 1. The samples were then reconstructed and processed with the CMS software version $C M S S W \_4 \_4 \_4$ and global tag $G R_{-} R_{-} 44 \_V 13$. The events are triggered using either two electron candidates with transverse momentum $p_{T}$ thresholds of 17 and $8 \mathrm{GeV}$ as well as loose shower shape and isolation cuts. We use the JSON file Cert_160404-

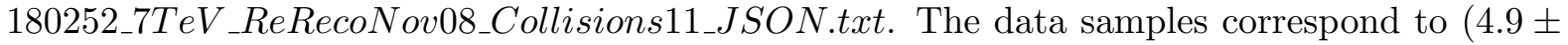
0.1) $\mathrm{fb}^{-1}$ of proton-proton collisions at $\sqrt{\mathrm{s}}=7 \mathrm{TeV}$.

All fully-reconstructed Monte Carlo (MC) samples in this analysis were produced during the "Summer2011" official production. MC Samples and corresponding cross-sections are detailed in Table 22, Both the MADGRAPH [5] and SHERPA [6] simulated samples contain zero to four jets at the matrix element, and were generated with a dilepton mass cut: $M>50 \mathrm{GeV} / \mathrm{c}^{2}$. We normalize the inclusive production cross section after this dilepton mass cut to the NNLO cross section predicted by FEWZ [7].

\section{Event Selection}

We remove events not coming from pp collisions, such as those from beam-gas interactions or beamscraping in the transport system near the interaction point, by requiring at least one good 
Table 3. The electron identification criteria for barrel and endcaps. Exact definition could be found in [8].

\begin{tabular}{|l|c|}
\hline Variable & Selections \\
\hline Algorithm & anti-kt \\
Size parameter R & $=0.5$ \\
Neutral Hadron Fraction & $<0.99$ \\
Neutral EM Fraction & $<0.99$ \\
Number of Constituents & $>1$ \\
Charged Hadron Fraction & $>0$ \\
Charged Multiplicity & $>0$ \\
Charged EM Fraction & $<0.99$ \\
\hline
\end{tabular}

Table 4. The particle-flow jet loose identification criteria. Exact definition could be found in 8.

\begin{tabular}{|l|c|c|}
\hline Variable & Barrel & Endcaps \\
\hline Supercluster $\eta_{S C}$ & $<1.4442$ & $1.566-2.1$ \\
$N_{\text {missinghits }}$ & $=0$ & $=0$ \\
A conversion & false & false \\
Combined relative PFisolation $\Delta R=0.4$ & $<0.2$ & $<0.2$ \\
$\sigma_{\text {inin }}$ & $<0.01$ & $<0.03$ \\
$\delta \phi_{\text {in }}$ & $<0.06$ & $<0.03$ \\
$\delta \eta_{\text {in }}$ & $<0.004$ & $<0.007$ \\
$H / E$ & $<0.04$ & $<0.15$ \\
\hline
\end{tabular}

primary interaction vertex to be reconstructed. Such vertices must have at least three tracks and must be within $15 \mathrm{~cm}(2 \mathrm{~cm})$ of the nominal center of the detector along (perpendicular to) the beam axis.

After these event selections, we require a pair of oppositely-charged particle-flow electron candidates to have an invariant mass within the $Z^{0}$ mass window and exactly one particleflow jet that satisfies the object identification and basic kinematic selection. We then study the angular correlation between this $Z^{0}$ candidate and this jet. Table 3 and Table 4 list the identification requirements for the electron and jet candidates, respectively [8].

For this analysis, exclusive $Z+1$ jet events are used to isolate the differences between data and theory. If another jet with $p_{T}>30 \mathrm{GeV}$ and $|\eta|<2.4$ exists, the event is rejected. To reconstruct jets and leptons, the particle flow $(\mathrm{PF})$ algorithm [9] is used, which attempts to identify all the stable particles in an event by using the full ensemble and redundancy of the CMS detector. Jets are reconstructed using the anti-kT algorithm [10] with a distance parameter of $R=0.5$ and are required to have $|\eta|<2.4$ to benefit from the tracker coverage. The energy of the charged particles coming from pileup interactions, not associated with the hard scattering, is subtracted using vertex information. The neutral energy from pileup events adds $0.5 \mathrm{GeV}$ per additional interaction to the jet energy, which is subtracted on an event by event basis. Dielectron events are required to have at least two oppositely-charged reconstructed electrons with $p_{T}>20 \mathrm{GeV},|\eta|<2.1$ and an invariant mass $\left(M_{e e}\right)$ in the range of $76-106 \mathrm{GeV}$ around the $\mathrm{Z}$ boson mass. We apply a minimum $p_{T}$ threshold on the $Z^{0}$ boson at $40 \mathrm{GeV} / \mathrm{c}$. Although the $p_{T}\left(Z^{0}\right)>40 \mathrm{GeV} / \mathrm{c}$ selection reduces the amount of events available at large $Y_{\text {diff }}$ and $Y_{\text {sum }}$, it allows a fair comparison with the photon channel [11.

Detailed explanation of electron reconstruction in CMS can be found in [12]. The combined relative isolation of a lepton is dened as the sum of electromagnetic and hadronic calorimeter energies, plus charged track momentum in a $\eta-\phi$ cone of radius 0.4 , divided by the lepton momentum. Electrons and jets are required to be separated $[R(e, j e t)>0.5]$ in $\eta-\phi$ space. After this selection the sample is $99 \%$ pure in $Z+1$ jet events. Small contributions from the processes $t \bar{t}$, diboson production, and from QCD processes are neglected.

\section{Results}

We compare the rapidity distributions for events with a $\mathrm{Z}$ boson in association with one jet between data and MC at the CERN Large Hadron Collider using the CMS detector. The protonproton collision data used in this analysis corresponds to an integrated luminosity of $4.9 \mathrm{fb}^{-1}$ at $\sqrt{s}=7 \mathrm{TeV}$. Rapidity distributions that are compared between data and MC are $: Y_{V}, Y_{\text {jet }}$, $Y_{\text {sum }}$ and $Y_{\text {dif }}$. Figure 1 shows the distributions from Data and sum of simulated MADGRAPH 

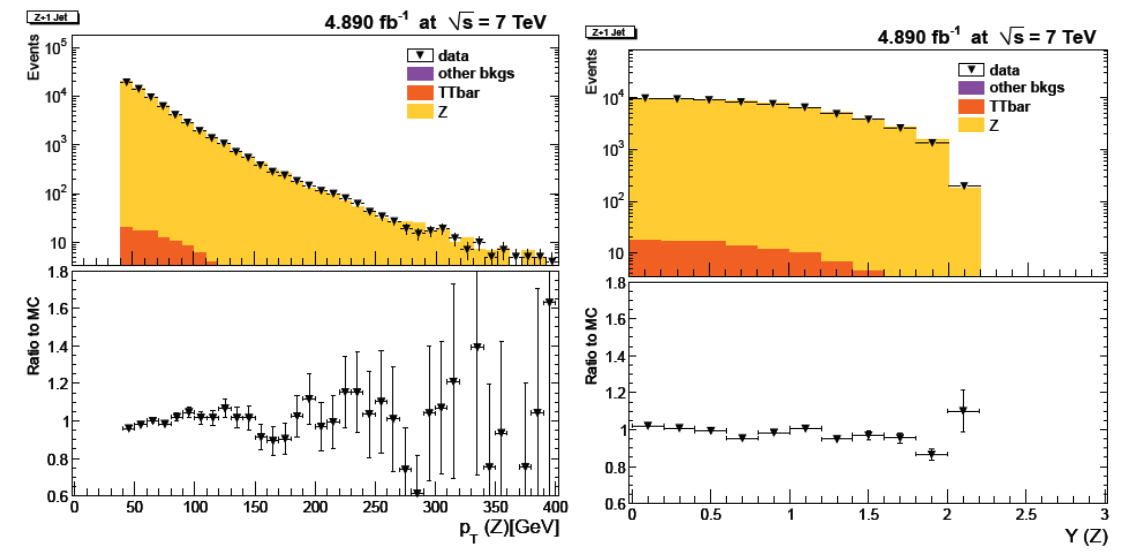

Fig. 1. Distributions from Data and from the sum of simulated MADGRAPH $Z^{0} / \gamma^{*}, t \bar{t}+j e t s$ and PYTHIA QCD events, for $p_{T}\left(Z^{0}\right)$ and $\left|Y_{z}\right|$.
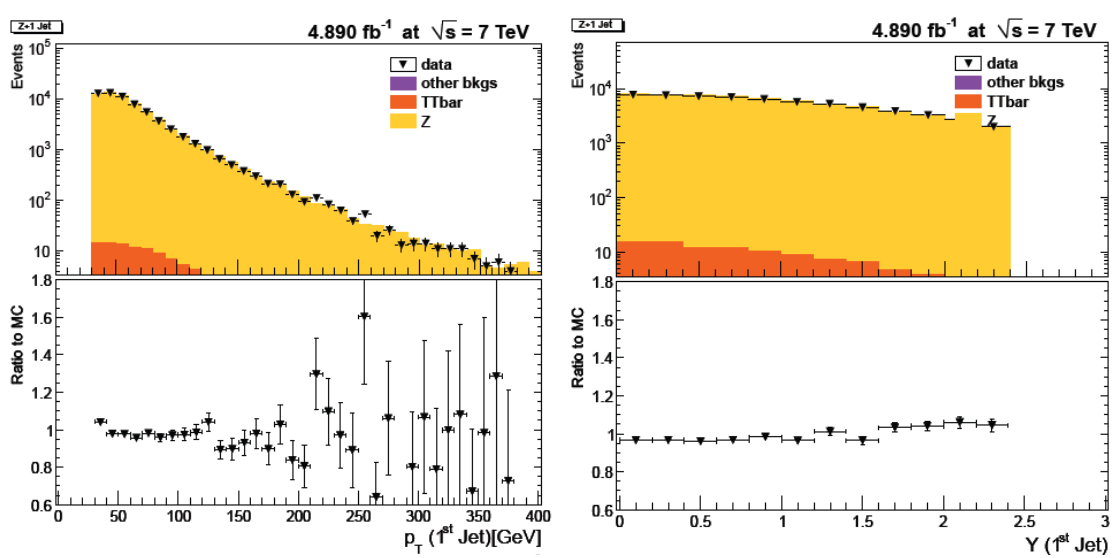

Fig. 2. Distributions from Data and from the sum of simulated MADGRAPH $Z^{0} / \gamma^{*}, t \bar{t}+j e t s$ and PYTHIA QCD events, for $p_{T}($ jet $)$ and $\left|Y_{\text {jet }}\right|$.
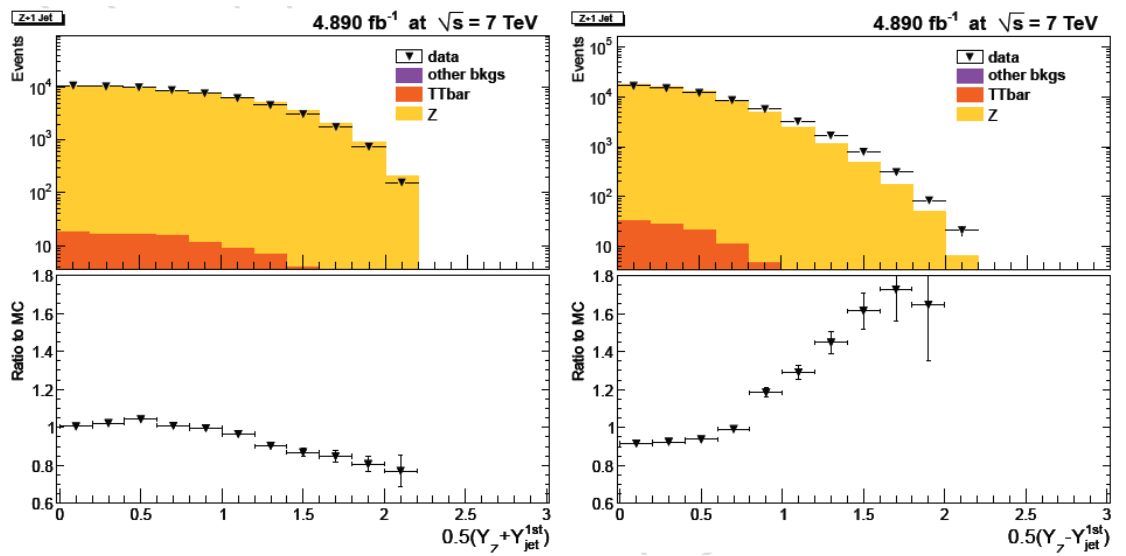

Fig. 3. Distributions from Data and from the sum of simulated MADGRAPH $Z^{0} / \gamma^{*}, t \bar{t}+j e t s$ and PYTHIA QCD events, for $\left|Y_{\text {sum }}\right|$ and $\left|Y_{\text {dif }}\right|$. 
$Z^{0} / \gamma^{*}, t \bar{t}+j e t s$ and PYTHIA QCD events, for $p_{T}\left(Z^{0}\right)$ and $\left|Y_{z}\right|$. Transverse Momentum and Rapidity distributions of Jet are shown in Figure 2 , $\left|Y_{\text {sum }}\right|$ and $\left|Y_{\text {diff }}\right|$ distributions from Data and $\mathrm{MC}$ are shown in Figure 3. Data and MC agreement is good as seen in Figures 1, 2 and 3. We will compare our measurements with a next-to-leading-order perturbative QCD calculation and two generator programs that combine tree-level matrix element calculations with parton showers.

\section{Conclusions}

We present the area-normalized distributions of the angular correlation in events containing $Z^{0} \rightarrow e e+$ Jet, using $4.9 \mathrm{fb}^{-1}$ of proton-proton collisions at $\sqrt{s}=7 \mathrm{TeV}$ collected by CMS in the year of 2011. Good agreement is found between data and MC. Our results are consistent with that of the muon channel [13].

\section{Acknowledgements}

We thank the technical and administrative staffs at CERN and at other CMS institutes for their contributions. We thank our collaborators A.P. Singh, S.S. Yu, D. Mekterovic,Y.H. Chang,W. Lin1 and L.K. Saini from National Central University, Chung-Li, Taiwan for this work. Financial support from UGC/DAE/DST is duly acknowledged.

\section{References}

[1] H. Ita et al., "Precise predictions for Z-boson + 4jet production at hadron colliders, Phys.Rev.D85 (2012) 031501(R), doi:10.1103/PhysRevD.85.031501.

[2] J. Owens, Large-momentum-transfer production of direct photons, jets, and particles, Rev. Mod. Phys. 59 (1987) 465, doi:10.1103/RevModPhys.59.465.

[3] Z. Belghobsi et al., Photon-jet correlations and constraints on fragmentation functions, Phys. Rev. D79 (2009) 114024, doi:10.1103/PhysRevD.79.114024.

[4] CMS Collaboration, The CMS experiment at the CERN LHC, JINST 3 (2008) S08004, doi:10.1088/17480221/3/08/S08004.

[5] J. Alwall, M. Herquet, F. Maltoni et al., "MadGraph 5:Going Beyond,JHEP 1106 (2011) 128, doi:10.1007/JHEP06(2011)128, arXiv:1106.0522.

[6] J. Archibald, T. Gleisberg, S. Hoeche et al.," Recent developments in SHERPA, Nucl.Phys.Proc.Suppl. 183 (2008) 6066.

[7] R. Gavin, Y. Li, F. Petriello et al., FEWZ 2.0: A code for hadronic Z production at next-to-next-to-leading order, Comput.Phys.Commun. 182 (2011) 23882403, doi:10.1016/j.cpc.2011.06.008, arXiv:1011.3540.

[8] L.K. Saini, A.P. Singh, D. Mekterovic, S.S. Yu, K. Mishra, S. Dasu, S.B. Beri, Measurement of $d \sigma\left(Z^{0}+n\right) / d p_{T}^{n t h J e t}$ and $d \sigma\left(Z^{0}+n\right) / d Y^{n t h J e t}$ in Proton-Proton Collisions at $\sqrt{s}=7 T e V$, CMS Analysis Note AN-12-033 (2012).

[9] CMS Collaboration, "Particle-Flow Event Reconstruction in CMS and Performance for Jets, Taus, and MET", CMS Physics Analysis Summary CMS-PAS-PFT-09-001 (2009).

[10] M. Cacciari, G. P. Salam, and G. Soyez, "The anti-kt jet clustering algorithm", JHEP 04 (2008) 063, doi:10.1088/1126-6708/2008/04/063.

[11] S.L. Linn, Y.J. Lu, V. Gaultney-Werner, S.S. Yu, "Angular Distribution of Photon+Jet Events in the Center-of-Mass Frame for $\sqrt{s}=7 \mathrm{TeV}$, CMS Analysis Note AN-11-497 (2012).

[12] CMS Collaboration, "Electron reconstruction and identication at $\sqrt{s}=7 \mathrm{TeV} "$, CMS Physics Analysis Summary CMS-PAS-EGM-10-004 (2010).

[13] L. Lebolo, S.L. Linn, K. Kovitanggoon, S.W. Lee and others, "Angular Distributions of $Z^{0}$ bosons in $Z^{0}+$ Jet Events Using 2011 Data, CMS Analysis Note AN-12-037 (2012). 